\title{
Numerical Simulations of the Light-Extraction Efficiency of LEDs on Sapphire Substrates Pattemed with Various Polygonal Pyramids
}

\author{
Hao Cui and Si-Hyun Park* \\ Department of Electronic Engineering, Yeungnam University, Gyeongsan 712-749, Korea
}

(Received August 7, 2014 : revised November 19, 2014 : accepted December 3, 2014)

\begin{abstract}
We report a numerical analysis of the light-extraction efficiency (LEE) of light-emitting diodes (LEDs) on patterned sapphire substrates (PSSs). We considered various $n$-sided, regular convex pyramids, where $n$ is an integer and $n \geq 3$. We then considered four cross sections: extruded, subtracted, truncated-extruded, and truncated-subtracted. Ray-tracing simulations were carried out with these polygonal pyramid patterns, and the dimensions of the patterns were systematically varied. Optimized pattern shapes were determined for large LEE. An extruded circular pyramid with a slant angle of $45^{\circ}$ was found to be the optimal patterned shape.
\end{abstract}

Keywords : Light-emitting diode, Light-extraction efficiency, Patterned sapphire substrate, Ray-tracing analysis

OCIS codes : (230.3670) Light-emitting diodes; (290.5890) Scattering stimulated; (350.4600) Optical engineering

\section{INTRODUCTION}

Light-emitting diodes (LEDs) are solid-state light sources that exploit electroluminescence in semiconductor materials to generate light. LEDs are semiconductor $p$ - $n$ junction diodes, from which light is emitted due to the optical recombination of electrons and holes. The wavelength of the emitted light is determined by the band gap of the semiconductor, and is given (in $\mu \mathrm{m}$ ) by $\lambda=1.24 / E_{g}$, where $E_{g}$ is the band gap (in eV). LEDs have attracted much attention as light sources for applications such as indicator lights on devices, traffic lights, automobile lighting, and indoor and outdoor lighting, including street lighting. This is due their low energy consumption, long lifetime, robustness, absence of a warm-up period, favorable controllability, and good color rendering. To include a wider range of applications, improvements in the efficiencies of LEDs are required.

The light-extraction efficiency (LEE) of LEDs is an important parameter. The LEE of an LED is defined as the ratio of the number of photons emitted into free space from an LED chip to the number of photons emitted from the active region inside the LED chip [1]. It is limited by total internal reflection (TIR) inside the LED semiconductor chip, which typically has a larger refractive index than the surrounding material.
When the light generated from the active region inside the semiconductor is incident upon the interface between the semiconductor and the surrounding space, if the angle of the emitted light exceeds the critical angle, TIR occurs and light is trapped inside the semiconductor, eventually being dissipated as heat. The LEE of an LED is typically small, because there is a large difference in refractive index between the semiconductor and the surrounding space [2]. For example, the refractive index of gallium nitride $(\mathrm{GaN})$ is 2.5, and the LEE of a GaN-based LED chip with a simple rectangular shape into free space is only $4 \%[1,2]$. Numerous approaches have been used to improve the LEE, including photon recycling [3], flip-chip configurations [4], resonant cavities [5], a transparent surface contact layer [6], sidewall treatment [7], surface roughening [8], photonic crystals [9], chip shaping [10], and patterned sapphire substrates (PSSs) [11-14]. PSSs employ arrayed patterns on the entire top surface of the sapphire that forms the LED substrate. Scattering and multiple reflection of the light inside the LED chip can be enhanced with these structures, breaking the TIR condition and therefore improving the LEE. The PSS can also serve to reduce the threading-dislocation density during the growth of LED epitaxial layers.

Monte Carlo ray-tracing simulations are one of the most

\footnotetext{
*Corresponding author: sihyun_park@ynu.ac.kr

Color versions of one or more of the figures in this paper are available online.
} 
popular optical simulation methods for the analysis of photonic devices [15-18]. Geometrical optical phenomena in photonic devices can be analyzed using this method, including ray scattering, absorption and transmission in the semiconductor layers, Fresnel losses of the media due to different refractive indices, and the randomly emitted light from the active region of an LED [19]. Although a number of studies of the numerical design of PSSs have been reported, a systematic study of polygonal pyramid patterns in PSSs has not yet been carried out. Polygonal pyramid patterns may be the most important shapes for use in PSSs, because they may be realized on the sapphire using only simple, two-dimensional (2D) photolithography patterning and conventional etching processes.

Here we describe numerical simulations for LEDs using the Monte Carlo ray-tracing method. We examined the LEE of LEDs using PSSs, focusing on PSSs with various polygonal pyramid patterns. By systematically varying the possible shapes of the polygonal pyramid patterns, we determined the pattern shape and size for optimal LEE.

\section{RAY-TRACING SIMULATION PROCEDURES}

We used the software application LightTools to implement ray-tracing simulations. Figure 1 shows a schematic diagram of the simulated GaN-based PSS-LED structure. The size

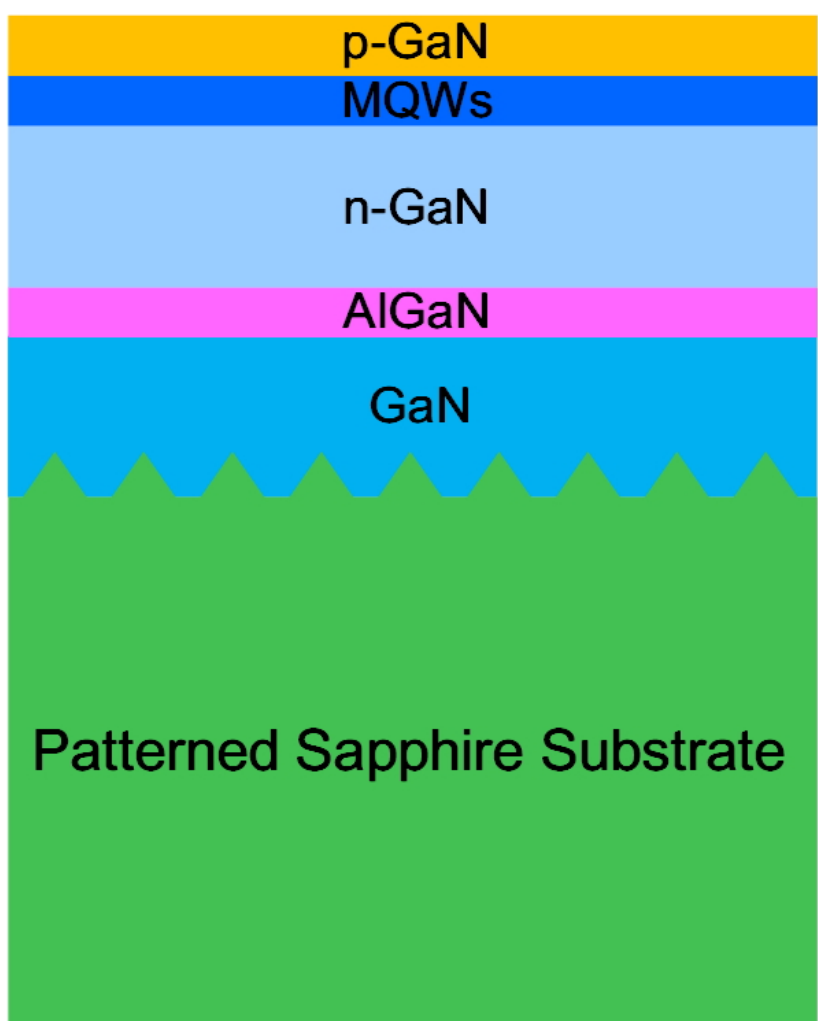

FIG. 1. Schematic diagram of the PSS-LED structure used in the ray-tracing simulations.
TABLE 1. Parameters for each layer in the ray-tracing simulations of the PSS-LEDs

\begin{tabular}{c|c|c|c}
\hline \hline \multicolumn{4}{|c}{ LED chip size : $300 \times 300 \mu \mathrm{m}^{2}$} \\
\hline layer & $\begin{array}{c}\text { thickness } \\
(\mu \mathrm{m})\end{array}$ & $\begin{array}{c}\text { refractive } \\
\text { index }\end{array}$ & $\begin{array}{c}\text { absorption } \\
\text { coefficient } \\
\left(\mu \mathrm{m}^{-1}\right)\end{array}$ \\
\hline$p$-GaN & 0.05 & 2.45 & 0 \\
\hline MQWs & 0.1 & 2.54 & 1 \\
\hline$n$-GaN & 2 & 2.42 & 0 \\
\hline$(\mathrm{Al}, \mathrm{Ga}) \mathrm{N}$ & 0.05 & 2.40 & 0 \\
\hline GaN & 2 & 2.40 & 0 \\
\hline Sapphire & 330 & 1.78 & 0 \\
\hline
\end{tabular}

of the chip was $300 \times 300 \mu \mathrm{m}^{2}$. The parameters for each layer in the PSS-LED structure are listed in Table 1. The device consisted of a 330- $\mu$ m-thick sapphire substrate, a 2$\mu \mathrm{m}$-thick GaN layer, a $0.05-\mu \mathrm{m}$-thick $(\mathrm{Al}, \mathrm{Ga}) \mathrm{N}$ layer, a 2$\mu \mathrm{m}$-thick $n$-type GaN layer, a $0.1-\mu \mathrm{m}$-thick multiple quantum well (MQW) layer, and a $0.05-\mu \mathrm{m}$-thick $p$-type GaN layer. The refractive indices of these layers were 1.78, 2.4, 2.4, $2.42,2.54$, and 2.45 respectively [20]. The absorption coefficient of the MQW layer was assumed to be $10^{4} \mathrm{~cm}^{-1}[19$, $21]$, and the reflectivity of bottom surface of the sapphire was $90 \%$ [22]. To simplify the LED structure, the mesa and electrode structures were neglected in our simulations, and the PSS patterns were modeled on the top surface of the sapphire substrate. We considered only an LED chip structure without a package (PKG) structure, and therefore the material outside is assumed to be simply air in our study. We think, however, our results without PKG can also be applied in general to LEDs with PKG, at the qualitative level.

We chose to consider only polygonal pyramid patterns, because these patterns can be expected to be fabricated reasonably on the sapphire substrate via simple control of the $2 \mathrm{D}$ etching mask pattern shape, in addition to the etching conditions. We did not consider pillars because the slant angle of the pillar pattern is fixed at $90^{\circ}$, and so trapped light cannot efficiently out-couple via multiple reflections.

We aimed to cover many possible shapes of the polygonal pyramid patterns. We considered pyramids with bases of $n$-sided regular convex polygons, where $n=3,4,6$, and $\infty$, (i.e., pyramids with triangular, square, hexagonal, and circular bases). Here, the pyramid pattern with a circular base is also known as a cone. Four different cross sections of the pyramids were considered: extruded, subtracted, truncatedextruded, and truncated-subtracted. The global arrangement of the patterns on the sapphire substrate was a regular hexagonal array (or honeycomb), which is widely used due to its high degree of integration. Figure 2 shows the different polygonal pyramid patterns that were investigated. To analyze and compare the LEE of the differently shaped PSSs as a function of the size of patterns, we considered the circumcircle 


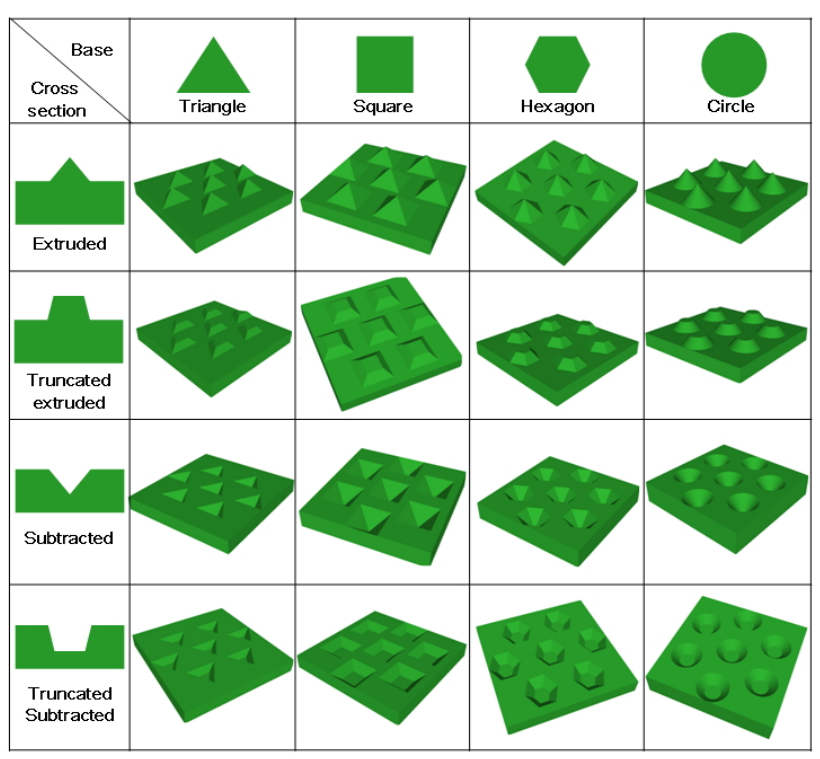

FIG. 2. The various polygonal pyramid patterns considered in this work.

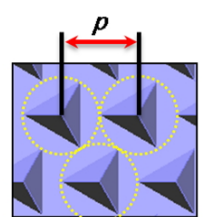

(a)

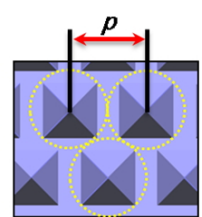

(b)

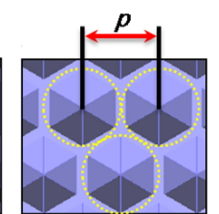

(c)

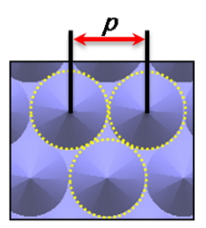

(d)
FIG. 3. The circumcircle of the base of the polygonal pyramids in close-packed PSS patterns. The dashed yellow lines are the circumcircles of the bases, and the red lines show the pitch $p$ of the patterns. (a) Triangular pyramid, (b) square pyramid, (c) hexagonal pyramid, and (d) circular pyramid.

diameter of the pyramid base. Figure 3 shows a plan-view of the array patterns of the four different bases. In the examples shown in the figure, the diameter of the circumcircle is the same as the pitch, i.e. the pattern is a close-packed array of the circumcircle. We independently varied the circumcircle diameter and the pitch in addition to the vertical height of the patterns, and then carried out simulations for each pattern. We defined the relative LEE of PSS-LED as the ratio of the LEE of PSS-LED to that of a reference LED, which did not have the PSS pattern. Using this normalized LEE, we determined the relationships between the LEE and the patterns used on the PSS.

\section{RESULTS AND DISCUSSION}

Figure 4 shows simulated relative LEEs of the PSS-LEDs with various polygonal pyramid patterns. We started with a pitch of $p=3.0 \mu \mathrm{m}$, which is typical of commercially used PSSs [23, 24]. We then varied the diameter of the

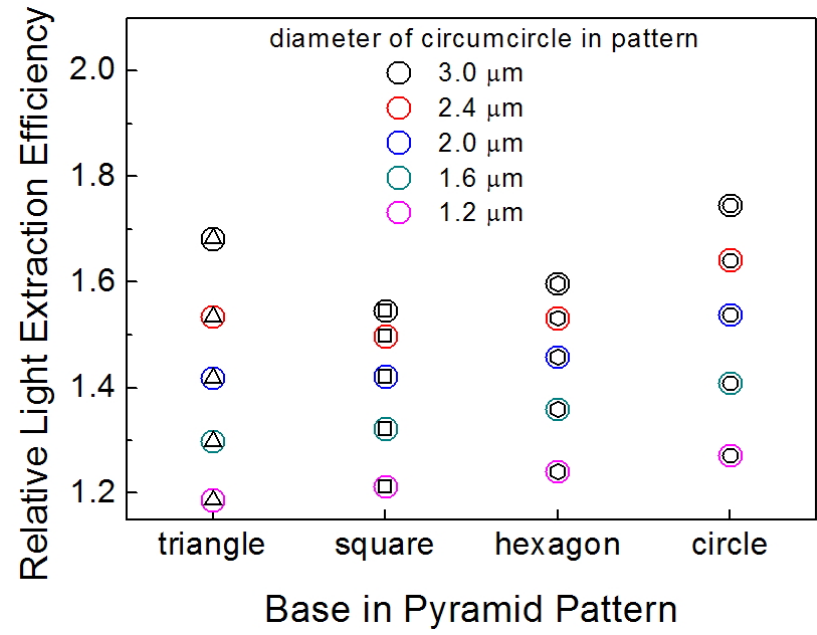

FIG. 4. The LEE for the various PSS-LEDs, relative to that of an LED without the PSS, for pyramids with various polygonal bases circumcircle diameters less than the pattern pitch of $3 \mu \mathrm{m}$, and pattern heights of one-half the diameter.

circumcircle in each pattern in the range $1.2 \leq d \leq 3.0 \mu$ $\mathrm{m}$, i.e. with $d$ in the range $0.4 \leq d / p \leq 1$. The height of the pattern was $h=d / 2$, so that the angle between slant edge and base was $45^{\circ}$. The four regular pyramid bases (i.e., $n=3,4,6$, and $\infty$ ) were independently considered, and the extruded type was considered for the cross section of the pyramid.

For each base shape, with a fixed pattern pitch, the LEE increased with the circumcircle diameter. In other words, the LEE increased as the fraction of the patterned surface area increased, and reached a maximum when the circumcircle diameter was at its maximum, i.e. $d=p$, a close-packed array. This result is to be expected, as one may anticipate that the scattering of the trapped light inside the structure will increase as a function of the fraction of the surface that is covered by the PSS patterns.

With a fixed circumcircle diameter, the circular-base pyramid exhibited a larger LEE than those with $n=3,4$, or 6. This is consistent with the above result, as the circular base has the largest proportion of patterned surface area. The hexagonal-base pyramid exhibited a larger LEE than the square-base pyramids; however, the triangular-base structures exhibited a larger LEE than the square- or hexagonalbase pyramids, despite the fact that the triangular structures had the largest proportion of unpatterned planar surface area. The $n=3$ and $n=\infty$ pyramids are therefore preferred. When the ratio with the circumcircle diameter remained fixed, so that we had a close-packed array of circumcircles (and the angle between the slant edge and the base was $45^{\circ}$ , and the proportion of unpatterned planar surface was also unchanged) the LEE did not change as a function of pattern pitch. It follows that we may have freedom of choice over the pattern height, so that we have flexibility in terms of the conditions for sapphire etching and epitaxy for the 


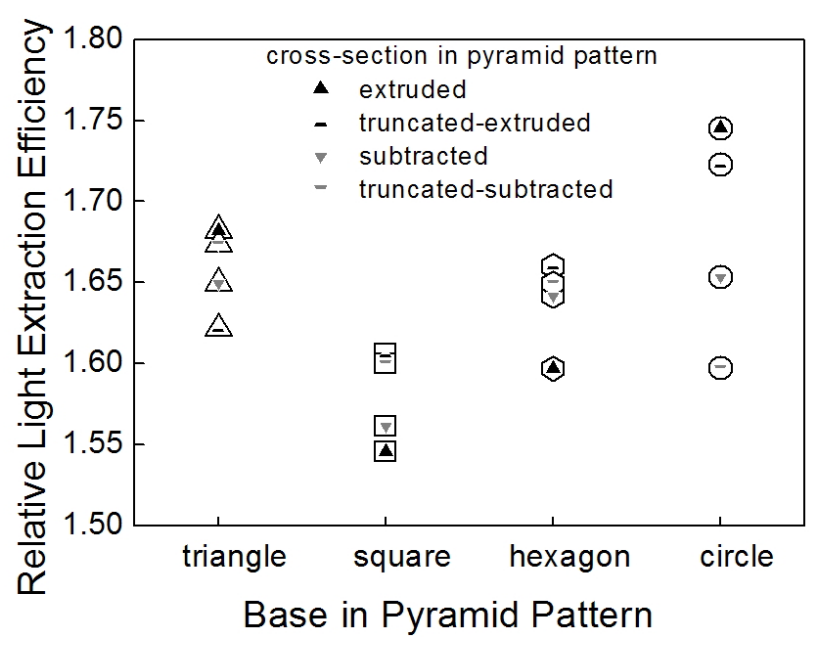

FIG. 5. The LEE for the different PSS-LEDs, relative to that of an LED without the PSS, for pyramids with various polygonal bases and cross-sectional shapes with a pitch of 3 $\mu \mathrm{m}$ and a circumcircle diameter of $3 \mu \mathrm{m}$.

growth of the LED structure.

Four cross-sectional shapes were considered: extruded, subtracted, truncated-extruded, and truncated-subtracted. We fixed the pattern pitch at $p=3.0 \mu \mathrm{m}$, and the diameter of the circumcircle at $d=p$. The vertical height of the pattern was also fixed, at $h=d / 2$ for the non-truncated types and $h=d / 4$ for the truncated shapes, so that the slant angles were fixed at $45^{\circ}$ in all cases. The LEE was calculated for the four regular, convex pyramid bases, as shown in Fig. 5 . The effect of the cross-sectional shape on LEE differed with base shape. The circular and triangular bases exhibited the largest LEE with the extruded cross section, followed by the truncated-extruded, the subtracted, and then the truncated-subtracted cross sections. For the square and hexagonal bases, the truncated-extruded cross section had the largest LEE, followed by the truncated-subtracted, the subtracted and then the extruded cross section. The LEE was largest with the circular base and the extruded cross section.

After determining the optimal geometry of the base and the cross section of the pyramid, we varied the vertical height, thereby varying the slant angle. Figure 6 shows the LEE with the extruded circular-base pyramid patterns as a function of pattern height, for various diameters of circumcircles with a pitch of $3.0 \mu \mathrm{m}$. For a given height of the pattern, the LEE increased with the diameter of the circumcircle. For a given circumcircle diameter, when $d \leq$ $2.5 \mu \mathrm{m}$, the LEE increased with the height of the pattern. In practical epitaxial growth, however, the height of the pattern should be limited to less than the thickness of the $n$-type GaN layer. For $d$ greater than about $2.5 \mu \mathrm{m}$, the pattern has the optimum height for a maximum LEE under the given circumcircle's diameter. For example, with $d=$ $3.0 \mu \mathrm{m}$ (a close-packed array of circumcircles), the LEE initially increased with increasing $h$, reached a maximum at $h=1.5 \mu \mathrm{m}$, and then decreased with further increase in $h$.

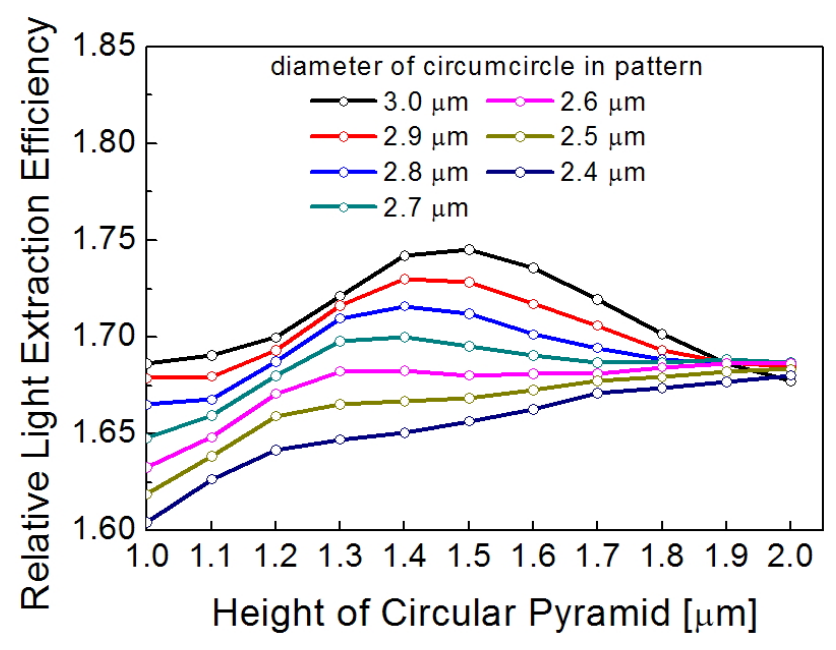

FIG. 6. The LEE for the various PSS-LEDs, relative to that of an LED without the PSS, for the extruded circular-base pyramid pattern, as a function of the pattern height for various circumcircle diameters, and with a pattern pitch of $3 \mu \mathrm{m}$.

Based on these results, we can conclude that the extruded cross section and circular-base pyramid pattern with $p=d$ $=h / 2$ (i.e. a slant angle of $45^{\circ}$ ) has the largest LEE. The actual physical values of $p, d$ and $h$ appear to be irrelevant, so long as the constraint $p=d=h / 2$ is observed, which provides some flexibility in terms of the epitaxial growth of the LED.

Additionally, we want to make the following points regarding the basic concern about the validity of the raytracing model for the calculation of LEE in PSS-LEDs with micron-sized structures. It is known that most researchers have successfully simulated light extraction in LEDs using the ray-tracing method when they considered LEDs with mixed structure sizes, i.e. some micron-sized structures and some submicron-sized, like our LEDs [14, 16, 17, 19, 20], even though they used a strict method such as the finitedifference time-domain (FDTD) method when they considered LEDs with entirely nanoscale structures. The validity for such a use of the ray-tracing method can be explained: The key point in the phenomenon of light extraction with PSSLEDs is the breaking of TIR, so that the light's nature as a ray is more conspicuously considered than as a wave. Recently, it has also been reported that simulation results for LEE enhancement of LEDs with entirely nanoscale structures are similar for ray-tracing and FDTD, the difference not exceeding $8.5 \%$ [18], which justifies our reasoning above.

\section{CONCLUSION}

We have described the results of Monte Carlo ray-tracing simulations of the LEE of PSS-LEDs with various polygonal pyramid patterns. We systematically varied the geometry of the polygonal pyramid patterns, and found relationships between the LEE of PSS-LEDs and the geometry of the 
patterns. The LEE increased as the fraction of the unpatterned planar surface area decreased. Triangular- and circular-base pyramids yielded a maximal LEE for an extruded cross section, whereas square- and hexagonal-bases had maximal LEEs for a truncated-extruded cross section. The LEE was largest for a circular base, followed by a triangular base. In general, the LEE increased with the height of the pattern when there was a large fraction of unpatterned planar surface. However, when the fraction of the unpatterned planar surface was below a certain critical value, the pattern exhibited an optimum height. The circular-base pyramid pattern with an extruded cross section, $p=d=h / 2$ (i.e. a slant angle of $45^{\circ}$ ), and a $2 \mathrm{D}$ close-packed hexagonal pattern had the largest LEE.

\section{REFERENCES}

1. E. F. Shubert, Light-Emitting Diodes, 2nd ed. (Cambridge University Press, Cambridge, UK, 2006).

2. A. Zukauskas, M. S. Shur, and R. Caska, Introduction to Solid-State Lighting (John Wiley \& Sons, New York, USA, 2002).

3. X. Guo, J. W. Graff, and E. F. Shubert, "Photon-recycling for high brightness LEDs," Comp. Semi. 6, 1-4 (2000).

4. J. J. Wierer, D. A. Steigerwald, M. R. Krames, J. J. O'Shea, M. J. Ludowise, G. Christenson, Y. C. Shen, C. Lowery, P. S. Martin, S. Subramanya, W. Gotz, N. F. Gardner, R. S. Kern, and S. A. Stockman, "High-power AlGaInN flip-chip light-emitting diodes," Appl. Phys. Lett. 78, 3379-3381 (2001).

5. D. Delbeke, R. Bockstaele, P. Bienstman, R. Baets, and H. Benisty, "High-efficiency semiconductor resonant-cavity lightemitting diodes: A review," IEEE J. Select. Topics Quantum Electron. 8, 189-205 (2002).

6. S. M. Pan, R. C. Tu, Y. M. Fan, R. C. Yeh, and J. T. Hsu, "Improvement of InGaN-GaN light-emitting diodes with surface-textured indium-tin-oxide transparent ohmic contacts," IEEE Photon. Technol. Lett. 15, 649-651 (2003).

7. C. F. Lin, Z. J. Yang, J. H. Zheng, and J. J. Dai, "Enhanced light output in nitride-based light-emitting diodes by roughening the mesa sidewall," IEEE Photon. Technol. Lett. 10, 2038-2040 (2005).

8. Y. J. Lee, H. C. Kuo, S. C. Wang, T. C. Hsu, M. H. Hsieh, M. J. Jou, and B. J. Lee, "Increasing the extraction efficiency of AlGaInP LEDs via n-side surface roughening," IEEE Photon. Technol. Lett. 17, 2289-2291 (2005).

9. D. H. Kim, C. O. Cho, Y. G. Roh, H. S. Jeon, Y. S. Park, J. H. Cho, J. S. Im, C. S. Sone, Y. J. Park, W. J. Choi, and Q. H. Park, "Enhanced light extraction from GaN-based light-emitting diodes with holographically generated two-dimensional photonic crystal patterns," Appl. Phys. Lett. 87, 203508 (2005).

10. S. E. Brinkley, C. L. Keraly, J. Sonoda, C. Weisbuch, J. S. Speck, S. Nakamura, and S. P. Denbaars, "Chip shaping for light extraction enhancement of bulk c-plane light-emitting diodes," Appl. Phys. Express 5, 032104 (2012).

11. J. H. Lee, J. T. Oh, Y. C. Kim, and J. H. Lee, "Stress reduction and enhanced extraction efficiency of GaN-based LED grown on cone-shape-patterned sapphire," IEEE Photon. Technol. Lett. 20, 1563-1565 (2008).

12. Y. K. Su, J. J. Chen, C. L. Lin, S. M. Chen, W. L. Li, and C. C. Kao, "Pattern-size dependence of characteristics of nitride-based LEDs grown on patterned sapphire substrates," J. Cryst. Growth 311, 2973-2976 (2009).

13. Y. T. Hsu, C. C. Yu, K. F. Huang, W. H. Lan, J. E. Huang, J. C. Lin, and W. J. Lin, "Improved output power of nitride-based light-emitting diodes with convex-patterned sapphire substrates," IEEE Photon. Technol. Lett. 24, 1686-1688 (2012).

14. Y. C. Lee, S. C. Yeh, Y. Y. Chou, P. J. Tsai, J. W. Pan, H. M. Chou, C. H. Hou, Y. Y. Chang, M. S. Chu, C. H. $\mathrm{Wu}$, and C. H. Ho, "High-efficiency InGaN-based LEDs grown on patterned sapphire substrates using nanoimprinting technology," Microelectron. Eng. 105, 86-90 (2013).

15. V. Zabelin, D. A. Zakheim, and S. A. Gurevich, "Efficiency improvement of AlGaInN LEDs advanced by ray-tracing analysis," IEEE J. Quantum. Elect. 40, 1675-1686 (2004).

16. E. H. Park, J. Jang, S. Gupta, L. Ferguson, C. H. Kim, S. K. Jeon, and J. S. Park, "Air-voids embedded high efficiency InGaN-light emitting diode," Appl. Phys. Lett. 93, 191103 (2008).

17. Z. Y. Liu, K. Wang, X. B. Luo, and S. Liu, "Precise optical modeling of blue light-emitting diodes by Monte Carlo ray-tracing," Opt. Express 18, 9398-9412 (2010).

18. J. W. Pan, P. J. Tsai, K. D. Chang, and Y. Y. Chang, "Light extraction efficiency analysis of GaN-based light-emitting diodes with nanopatterned sapphire substrates," Appl. Opt. 52, 1358-1367 (2013).

19. J. W. Pan and C. S. Wang, "Light extraction efficiency of GaN-based LED with pyramid texture by using ray path analysis," Opt. Express 20, A630-A640 (2012).

20. T, X. Lee, K. F. Gao, W. T. Chien, and C. C. Sun, "Light extraction analysis of GaN-based light-emitting diodes with surface texture and/or patterned substrate," Opt. Express 15, 6670-6676 (2007).

21. A. B. Djurisic, Y. Chan, and E. H. Li, "Calculations of the refractive index of A1GaN/GaN quantum well," Proc. SPIE 4283, 630-637 (2001).

22. http://refractiveindex.info

23. http://www.rubicontechnology.com/sites/default/files/pdfs/pss.pdf

24. http://www.kqtech.co.kr/kor/02/product01.php 